\title{
The Seven Best from PSOGI 2016
}

\author{
Paul H. Sugarbaker, MD, FACS, FRCS \\ Center for Gastrointestinal Malignancies, MedStar Washington Hospital Center, Washington, DC
}

The Peritoneal Surface Oncology Group International (PSOGI) has met every other year for the last 20 years in order to provide a forum for progress in the prevention and treatment of peritoneal metastases. The 10th Biennial Meeting was held in Washington, DC, at the Omni Shoreham Hotel, with a total of 704 delegates, including 67 speakers. Fifty-four countries were represented among the participants at the meeting. A total of 325 abstracts were submitted and 274 posters were exhibited at the poster sessions. The awards banquet on Friday evening, 19 November 2016, was attended by 538 persons. Frans A.N. Zoetmulder, MD, PhD, and Francois N. Gilly, MD, PhD, received lifetime achievement awards.

The historical video presented at the 'Awards Banquet' highlighted the PSOGI conferences that have been held to date, the city that hosted the meeting, and the number of participants registered to attend (Table 1). The meeting has grown from a small number of participants centered in just a few institutions around the world into a global interest group with participants from surgery, medical oncology, radiology, and pathology. A strong representation of nurses and other allied healthcare professionals regularly attend the biennial meeting.

The PSOGI conference for 2018 is scheduled to occur in Paris, France (Fig. 1 provides information pertaining to this 2018 meeting).

(C) Society of Surgical Oncology 2017

First Received: 17 January 2017;

Published Online: 6 February 2017

P. H. Sugarbaker, MD, FACS, FRCS

e-mail: Paul.Sugarbaker@medstar.net

\section{CLINICAL TRIALS AND MULTI-INSTITUTIONAL REGISTRIES REGARDING CYTOREDUCTIVE SURGERY/HYPERTHERMIC INTRAPERITONEAL CHEMOTHERAPY (CRS/HIPEC) IN THE PREVENTION AND TREATMENT OF PERITONEAL METASTASES}

Currently, there are numerous clinical studies, including randomized controlled trials, that are seeking to determine the role of cytoreductive surgery (CRS) plus hyperthermic intraperitoneal chemotherapy (HIPEC) in the management of gastrointestinal and gynecologic malignancy. Current clinical trials that are active or awaiting the maturation of results after the trial had been closed are shown in Table 2, including proactive colorectal cancer protocols, colorectal treatment protocols, ovarian cancer protocols, and gastric cancer protocols. The protocol, presenter, and country of origin of the trial are included in this table.

Another multi-institutional effort in the studies of peritoneal surface oncology are the international and national registries. The International Registry on Peritoneal Mesothelioma has accumulated data on 1078 peritoneal mesothelioma patients from 34 different institutions. Big RENAPE collecting data on rare diseases treated by CRS and HIPEC has accumulated 781 patients from 53 centers worldwide. The group in Sydney, Australia, accumulated data on repeat CRS in 231 patients from 13 institutions. Yan $\mathrm{Li}$ and colleagues accumulated data on CRS and HIPEC for peritoneal metastases from small bowel adenocarcinoma on 177 patients from 21 institutions worldwide. The German registry now includes 3404 patients (Table 3).

A national registry for The Netherlands has accumulated data on 781 colorectal and appendiceal synchronous peritoneal metastases patients who were treated with CRS and HIPEC from 2005 to 2016. Aditi Bhatt from Bangalore, India, presented the Indian experience with 17 patients in a new nationwide CRS and HIPEC registry; this national 
TABLE 1 The historical video at the 'Awards Banquet' highlighted the date of PSOGI conferences, location, and attendance numbers

Summary of PSOGI biennial congresses

1998-Basingstoke (20 participants)

2000-Royal College of Surgeons Millenium Masterclass, London (30 participants)

2002-Basingstoke (50 participants)

2004-Madrid (250 participants) [special issue of EJSO]

2006-Uppsala (450 participants)

2008-Milan-Delphi method consensus conference (450 participants) [special issue of JSO]

2010-Lyon (450 participants) [special issue of The Cancer Journal]

2012-Berlin (600 participants) [spectacular dance party]

2014-Amsterdam (500 participants)

2016-Washington (700 participants)

2018-Paris, 6-8 September 2018, Maison de la Chimie, France

PSOGI Peritoneal Surface Oncology Group International, EJSO european journal of surgical oncology, JSO journal of surgical oncology

registry can serve as a model for other national registries to be initiated around the globe. There is no doubt that these multi-institutional registries have markedly increased our fund of knowledge regarding specific uses of CRS and HIPEC

A satellite symposium was held regarding the pharmacology of chemotherapy treatments for peritoneal metastases, with the goal of optimizing and standardizing the HIPEC regimens used around the globe. The indications and chemotherapy solutions for oxaliplatin-based chemotherapy and mitomycin C-based chemotherapy for colon cancer and appendiceal malignancy are presented in Table 4. In addition, a standardized HIPEC cisplatin treatment for front-line as well as platinum-sensitive and platinum-resistant recurrence of ovarian cancer is shown. Chemotherapy regimens recommended for small-volume peritoneal metastases from primary gastric cancer (Peritoneal Cancer Index [PCI] less than 6) and for mesothelioma and sarcoma were presented. Some rare diseases were recommended for treatment by HIPEC melphalan or gemcitabine. Of special note are the HIPEC gemcitabine protocols currently active for resected primary adenocarcinoma of the pancreas.

Another new trend for the management of peritoneal surface malignancy relates to the utilization of long-term intraperitoneal chemotherapy administered through an intraperitoneal port, often referred to as normothermic intraperitoneal chemotherapy long-term (NIPEC-LT). John Chan presented data from the Gynecologic Oncology Group pertaining to long-term intravenous and intraperitoneal chemotherapy protocols in ovarian cancer. Francois Quenet of Montpelier, France, talked about neoadjuvant intraperitoneal and systemic chemotherapy for colorectal peritoneal metastases with a PCI greater than 17. Hironori Ishigami presented data from Tokyo University on neoadjuvant intraperitoneal and systemic chemotherapy with paclitaxel for gastric cancer with peritoneal metastases. In the satellite symposium, Jenny Hong reviewed the rationale for combined long-term intraperitoneal and intravenous chemotherapy for ovarian cancer. Yutaka Yonemura commented on his mature data on intraperitoneal docetaxel and intraperitoneal cisplatin followed by CRS and HIPEC in patients who have gastric cancer peritoneal metastases and responded to the neoadjuvant intraperitoneal and systemic chemotherapy regimen. Results on long-term intraperitoneal and intravenous chemotherapy in malignant peritoneal mesothelioma was presented by Paul Sugarbaker. In summary, there is an expanding number of reports suggesting improved outcomes with NIPEC-LT. The inconvenience of the intraperitoneal port may have been surpassed by its efficacy reported at PSOGI 2016. The positive results are in high-grade cancers that have peritoneal metastases as a prominent part of their natural history.

An exciting new application of preoperative $\mathrm{CT}$ in patients with colon cancer was presented by Gina Brown of the Royal Marsden NHS Foundation Trust, London, UK. Her hypothesis is that the T-stage of colon cancer drives lymph nodal, hematogenous, and peritoneal dissemination. Accurate radiologic assessment preoperatively of the $T$ stage may help guide in the selection of patients for appropriate and individualized adjuvant treatments prior to, at the time of, or after colon cancer resection. Neoadjuvant chemotherapy or adjuvant HIPEC for colon cancers recognized as $\mathrm{T} 4$ by $\mathrm{CT}$ are alternatives to be considered. The commitment of the Peritoneal Surface Oncology Group in the prevention of colon cancer peritoneal metastases is emphasized by this work.

New data presented by John Spiliotis of Athens, Greece, and Naoual Bakrin of Lyon, France, suggest that the treatment of recurrent ovarian cancer for both cisplatinsensitive and cisplatin-resistant disease should be HIPEC cisplatin. The Big RENAPE group recommends cisplatin at $80 \mathrm{mg} / \mathrm{m}^{2}$ for $1 \mathrm{~h}$ at $42.5^{\circ} \mathrm{C}$ using a closed HIPEC. 

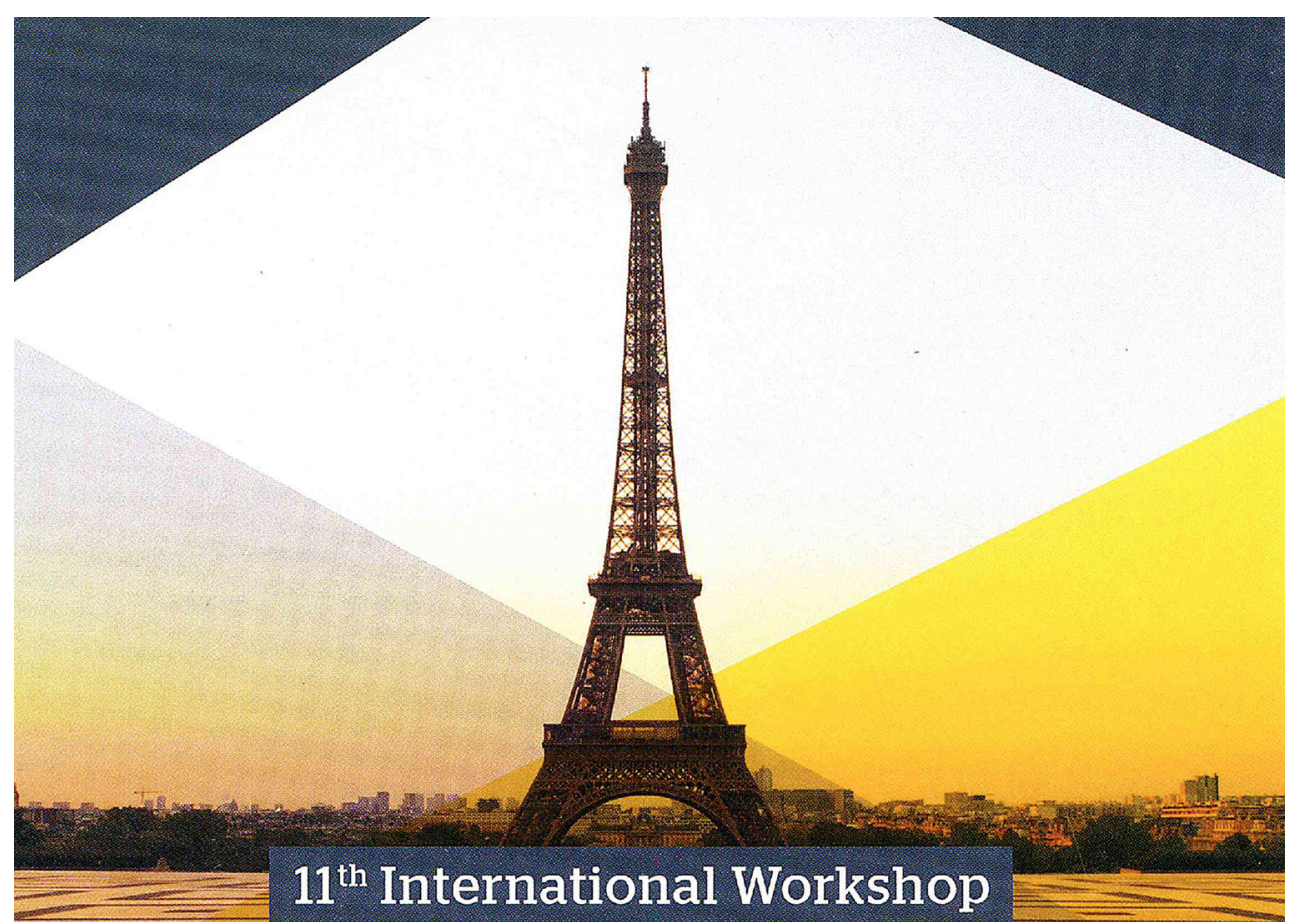

ON PERITONEAL SURFACE MALIGNANCY

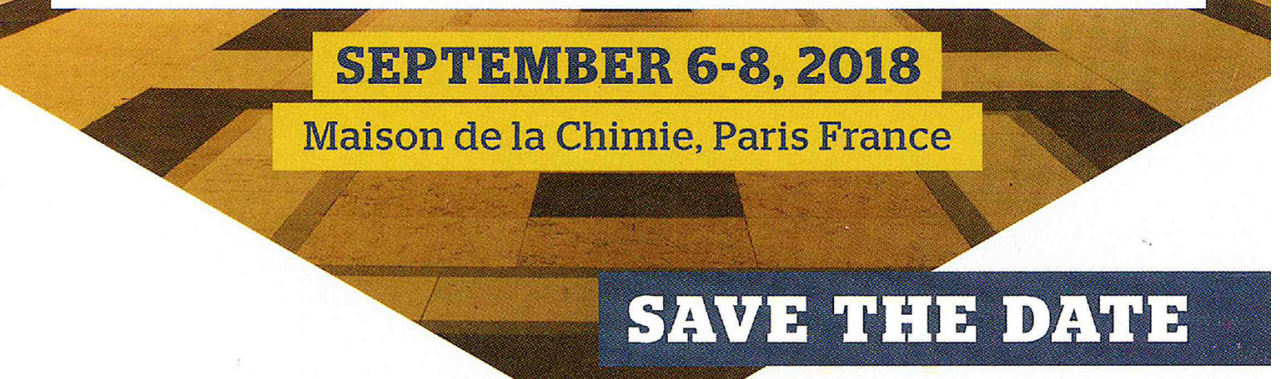

LOCAL

ORGANISING

COMIMITTER

Diane GOERE

Olivier GLEHEN

Marc POCARD

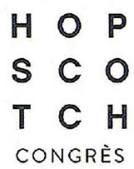

WWW.psogi2018.com

CONGRES

FIG. 1 Information regarding the Peritoneal Surface Oncology Group International Congress in 2018

Spiliotis and Bakrin presented results showing comparable survival with CRS plus HIPEC platinum for both cisplatinsensitive and cisplatin-resistant recurrent ovarian cancer (Table 5).

In conclusion, the take home messages from PSOGI 2016 were as follows:

1. Attend PSOGI 2018 in Paris, 6-8 September 2018.
2. Clinical trials testing HIPEC in colorectal cancer (ten trials), gastric cancer (three trials), and ovarian cancer (eight trials) are currently in progress.

3. Multi-institutional registries concerning the use of HIPEC (seven registries) have produced confirmatory useful data regarding the application of CRS and HIPEC. 
TABLE 2 Clinical trials testing cytoreductive surgery and hyperthermic intraperitoneal chemotherapy in gastrointestinal and gynecologic cancer

Proactive colorectal protocols

ProphyloCHIP: CRS and HIPEC with second-look surgery (Diane Goere, MD, PhD; Paris, France)

COLOPEC \pm adjuvant HIPEC for primary colon cancer (Pieter Tanis, MD, PhD; Amsterdam, The Netherlands)

PROMENADE: prophylactic HIPEC for primary colon cancer (Paolo Sammartino, $\mathrm{MD}, \mathrm{PhD}$; Rome, Italy)

Prophylactic: HIPEC for cT4 colon cancer (Alvaro Arjona-Sanchez, MD, PhD; Cordoba, Spain)

Colorectal treatment protocols

PRODIGE $7 \pm$ adjuvant HIPEC for peritoneal metastases from colon cancer (Francois Quenet, MD; Montpelier, France)

ICARUS EPIC FUDR versus HIPEC after optimal cytoreductive surgery (CRS) for neoplasms of the appendix, colon or rectum with isolated peritoneal metastasis (Garrett M. Nash, MD, MPH; New York City, NY, USA)

Surgery and oxaliplatin or mitomycin C in treating patients with tumors of the appendix (Edward A. Levine, MD; Winston-Salem, NC, USA)

Cytoreduction and intraperitoneal chemotherapy versus systemic chemotherapy in colorectal peritoneal carcinomatosis (Peter H. Cashin, $\mathrm{MD}, \mathrm{PhD}$; Uppsala, Sweden)

COMBATAC: combined anticancer treatment of advanced colon cancer (Pompiliu Piso, MD, PhD; Regensburg, Germany)

NIPOX adjuvant intraperitoneal oxaliplatin for colorectal cancer with peritoneal metastases (Francois Quenet, MD; Montpelier, France)

Ovarian cancer protocols

CRS and HIPEC in recurrent ovarian cancer (John D. Spiliotis, MD, PhD; Athens, Greece)

HIPEC with carboplatin for recurrent ovarian cancer, phase II (Dennis S. Chi, MD; New York City, NY, USA)

CHIPOR CRS with or without HIPEC for relapsed ovarian cancer (Jean-Marc Classe, MD, PhD; Nantes, France)

CHORINE CRS with or without HIPEC upfront for primary ovarian cancer (Luca Ansaloni, MD; Bergamo, Italy)

HORSE CRS with or without HIPEC in ovarian cancer recurrence (Anna Fagotti, MD, PhD; Rome, Italy)

Secondary debulking surgery \pm hyperthermic intraperitoneal chemotherapy in stage III ovarian cancer (Willemien van Driel, MD, PhD; Amsterdam, The Netherlands)

Outcomes in CRS/HIPEC as initial treatment of ovarian, fallopian tube, and primary peritoneal cancer (Teresa Diaz-Montes, MD;

Baltimore, MD, USA)

HIPEC in primary ovarian cancer, NCC Korea (Sang Yoon Park, MD, PhD; Seoul, Korea)

Gastric cancer protocols

GastriCHIP gastrectomy \pm HIPEC as adjuvant for primary gastric cancer (Olivier Glehen, MD, PhD; Lyon, France)

GASTRIPEC cytoreductive surgery (CRS) with/without HIPEC in gastric cancer with peritoneal carcinomatosis (Beate Rau, MD, PhD; Berlin, Germany)

Phoenix GC neoadjuvant intraperitoneal and systemic chemotherapy (NIPS) for GC with peritoneal metastases (Hironori Ishigami, MD; Tokyo, Japan)

TABLE 3 International and national multi-institutional registries reporting data on peritoneal surface malignancies (Presenter at PSOGI 2016 Congress)

International registry on peritoneal mesothelioma (Shigeki Kusamura, $\mathrm{MD}, \mathrm{PhD}$; Milan, Italy)

Big RENAPE including rare diseases treated with CRS and HIPEC (Diane Goere, MD, PhD; Paris, France)

Repeat cytoreductive surgery and hyperthermic intraperitoneal chemotherapy in colorectal cancer patients [PSOGI collaboration] (Nayef Alzahrani, MD; Sydney, NSW, Australia)

Cytoreductive surgery and hyperthermic intraperitoneal chemotherapy for peritoneal metastases from small bowel adenocarcinoma: multiinstitutional experience (Yang Liu, MD; Beijing, China)

German registry (Pompiliu Piso, MD, PhD; Regensburg, Germany)

Dutch registry (Vic Verwaal, MD, PhD; Aarhus, Denmark)

Indian HIPEC registry: a registry for indian patients with peritoneal surface Malignancies (Aditi Bhatt, MD, Mch; Bangalore, India) 
TABLE 4 Standardized hyperthermic intraperitoneal chemotherapy regimens presented at the Satellite Symposium on Intraperitoneal Chemotherapy, PSOGI 2016

\begin{tabular}{|c|c|c|}
\hline Indication & Chemotherapy solution & Duration (min) \\
\hline \multirow[t]{3}{*}{$\begin{array}{l}\text { Colorectal and appendiceal cancer } \\
\text { (prevention and treatment) }\end{array}$} & $\begin{array}{l}\text { IV 5-fluorouracil at } 400 \mathrm{mg} / \mathrm{m}^{2} \text { and IP oxaliplatin at } \\
460 \mathrm{mg} / \mathrm{m}^{2} \text { (open) }\end{array}$ & 30 \\
\hline & $\begin{array}{l}\text { IV 5-fluorouracil at } 400 \mathrm{mg} / \mathrm{m}^{2} \text { and IP oxaliplatin at } \\
360 \mathrm{mg} / \mathrm{m}^{2} \text { (closed) }\end{array}$ & 30 \\
\hline & $\begin{array}{l}\text { IP mitomycin } \mathrm{C} \text { at } 25-35 \mathrm{mg} / \mathrm{m}^{2} \text { (double or triple } \\
\text { dosed) }\end{array}$ & 90 \\
\hline $\begin{array}{l}\text { Ovarian cancer (front-line, platin- } \\
\text { sensitive or resistant recurrence) }\end{array}$ & Cisplatin $80 \mathrm{mg} / \mathrm{m}^{2}$ & 60 \\
\hline $\begin{array}{l}\text { Gastric cancer/peritoneal mesothelioma/ } \\
\text { sarcoma }\end{array}$ & Cisplatin $50 \mathrm{mg} / \mathrm{m}^{2}$ and doxorubicin $15 \mathrm{mg} / \mathrm{m}^{2}$ & 90 \\
\hline $\begin{array}{l}\text { Repeat HIPEC, unusual diseases, } \\
\text { progression on neoadjuvant } \\
\text { chemotherapy }\end{array}$ & Melphalan $60 \mathrm{mg} / \mathrm{m}^{2}$ & 60 \\
\hline Hepatobiliary, resected pancreas & Gemcitabine $1000 \mathrm{mg} / \mathrm{m}^{2}$ & 90 \\
\hline
\end{tabular}

PSOGI Peritoneal Surface Oncology Group International, HIPEC hyperthermic intraperitoneal chemotherapy, IV intravenous, IP intraperitoneal

TABLE 5 Data from Athens and France suggesting that hyperthermic intraperitoneal cisplatin is effective for cisplatin-sensitive and also cisplatin-resistant recurrent ovarian cancer

$\begin{array}{ll}\text { HIPEC [no. of } & \text { Median } \\ \text { patients }(\%)] & \text { survival } \\ & \text { (months) }\end{array}$

\begin{tabular}{lll}
$\begin{array}{l}\text { Athens } \\
\text { Platinum responsiveness }\end{array}$ & \\
$\quad$ Sensitive & $38(63.3)$ & 27.28 \\
Resistant & $22(36.7)$ & 26.08 \\
France & & \\
Platinum responsiveness & & \\
$\quad$ Sensitive & $246(52.4)$ & 47.2 \\
Resistant & $223(47.5)$ & 51.6 \\
\hline
\end{tabular}

HIPEC hyperthermic intraperitoneal chemotherapy
4. Guidelines for the chemotherapy regimens recommended for safe and effective HIPEC are now available.

5. An intraperitoneal port for long-term intraperitoneal or intraperitoneal and intravenous chemotherapy are currently being pursued (five studies reported).

6. The T stage of colon cancer can be estimated by CT, with profound implications for the management of primary disease.

7. HIPEC with cisplatin is active for cisplatin-resistant as well as cisplatin-sensitive recurrent ovarian cancer.

DISCLOSURE Paul Sugarbaker has no disclosures to declare. 